\section{Using Fluorochromes To Label An Oil-In-Water Emulsion}

Richard Thrift, SkyePharma, Inc

Richard_Thrift@SkyePharma.com

A question was asked on the microscopy listserver about fluorescent probes for studying oil-in-water emulsions trapped in a cellulose membrane. I don't have experience with that exact problem (I have studied chloroform/water emulsions rather than emulsions of triglycerides or alkanes), but I can make some general suggestions. The best type of hydrophobic probe to use depends on whether the probe is to be added to the hydrophobic phase before emulsification or after. If it is to be added to the preexisting emulsion as a dimethylsulfoxide stock solution, a probe with some aqueous solubility is required. Nile red and most small hydrophobic dyes have enough aqueous solubility to distribute through the water phase to the oil droplets. If this is to be done with a sample which contains a nitrocellulose filter membrane however, I expect there may be a problem with the probe transferring to the membrane and binding to hydrophobic sites there as well, adding to the background signal on the filter. Nile red has an advantage in that molecules in a polar environment do not fluoresce; the disadvantage is that it photobleaches rapidly. The hydrophobic pyrromethene dyes (such as BODIPY ${ }^{\text {B }} 493 / 503$ a.k.a. pyrromethene 546, BODIPY 505/515, and BODIPY 665/676) are much brighter and much more photostable, and are worth the expense in my experience. The pyrromethene dyes are said to be more specific than Nile Red, I presume because they are more hydrophobic. I haven't tried octadecylrhodamine B, Dil, etc.

If background on the membrane continues to be a problem, I would suggest a very hydrophobic probe such as a pyrromethene-labeled cholesterol ester or some other long alkylated or acylated nonpolar fluorophore. A Bodipy-labeled fatty acid, being polar, would not be the best choice. This would have to be added to the oil before emulsification. As the Mo-

\section{Adobe Photoshop ${ }^{\circledR} 6.0$ Distilled: Preparation of Micrographs for Publication Continued from preceding page}

- Save in the .PSD format to be able to edit or remove your scale bar, arrows, or text later.

\section{Finish up}

You should now have a beautiful figure plate! Make sure it is saved in the .PSD format, then you may save it as well in a TIFF format to send to the publisher. Photoshop 6.0 will automatically rasterize the text and shapes when selecting the TIFF format, but will maintain the layers. It will also automatically flatten the image (compressing all your layers into the background) for formats other than .PSD or TIFF, so those layers may not be independently edited again. If you need to make any changes to any of the individual images, shapes or text you will be able to open the .PSD version again to easily edit.

These steps in Photoshop are generally considered to be acceptable for the adjustment of micrographs for scientific presentation. Additional manipulations, such as Dodge and Burn may perhaps be used with restraint, but the use of Filters, the Cloning Tool and many other options may range from inappropriate to downright fraudulent. Remember, each of the pixels in an image contributes to your data! lecular Probes catalog points out for labeled cholesterol esters, such probes are basically nontransferable through water, and so shouldn't bind to the membrane unless the labeled emulsion wets it. But for the same reason, they will not label oil droplets uniformly if they are added as a dimethylsulfoxide stock solution to a preexisting emulsion.

If one wants to get fancy, one can ry using a rhodamine- or pyrromethene-labeled phospholipid to label the aqueous/organic interface (again, the probe should be added before emulsification in order to get uniform labeling). This makes beautiful pictures, especially with an oil phase counterstain of a contrasting color like the far-red pyrromethene Bodipy 665/676. Phosphatidylcholine or ethanolamine derivatives usually aren't soluble in the oil phase; I haven't tried Dil etc., and don't know whether the latter would also label the oil phase. Also, for striking contrast between the phases, the aqueous phase can be specifically labeled with hydrophilic probes such as dextran conjugates or (much better, in my experience) pyrromethene 556 (BODIPY $492 / 515$ disulfonate).

One concern, which may be minor for many users, is that the pyrromethene dyes themselves label the oil-water interface somewhat more brightly than the bulk oil phase. Apparently, the more nonpolar the oil phase (e.g., hexane), the more this effect is seen. To avoid the problem in such cases, the polarity of the probe should match that of the hydrophobic phase. Diphenylhexatriene is one possibility, although care must be taken to minimize photobleaching. Walter Niles has also suggested benzene (requiring $200 \mathrm{~nm}$ excitation) and decenene. Still, in many cases the pyrromethene probes will be quite suitable as a visual marker for the hydrophobic phase.

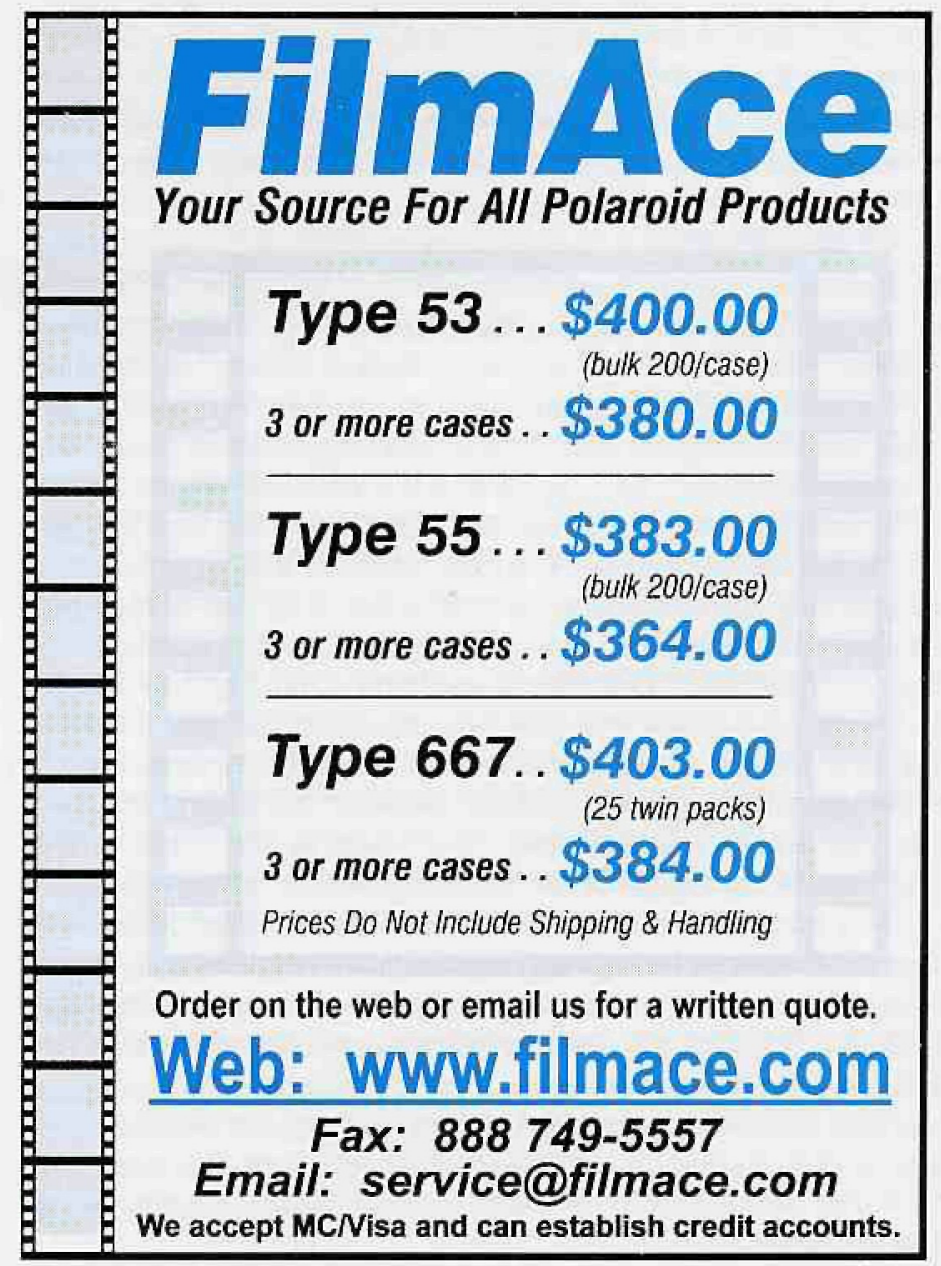

